\title{
What can we learn about Gribov copies from a formulation of QCD in terms of gauge-invariant fields?
}

\author{
Kurt Haller \\ Department of Physics, University of Connecticut, Storrs, CT 06269
}

\begin{abstract}
We review the procedure by which we implemented the non-Abelian Gauss's law and constructed gauge-invariant fields for QCD in the temporal (Weyl) gauge. We point out that the operator-valued transformation that transforms gaugedependent temporal-gauge fields into gauge-invariant ones has the formal structure of a gauge transformation. We express the "standard" Hamiltonian for temporalgauge QCD entirely in terms of gauge-invariant fields, calculate the commutation rules for these fields, and compare them to earlier work on Coulomb-gauge QCD. We also discuss multiplicities of gauge-invariant temporal-gauge fields that belong to different topological sectors and that, in previous work, were shown to be based on the same underlying gauge-dependent temporal-gauge fields. We relate these multiplicities of gauge-invariant fields to Gribov copies. We argue that Gribov copies appear in the temporal gauge, but not when the theory is represented in terms of gauge-dependent fields and Gauss's law is left unimplemented. There are Gribov copies of the gauge-invariant gauge field, which can be constructed when Gauss's law is implemented.
\end{abstract}

\section{Introduction}

Quantum Chromodynamics can be quantized in the temporal (or Weyl) gauge (in which $A_{0}^{a}=0$ ), in a number of ways. When the gauge-fixing term $\mathcal{L}_{\text {gauge }}=$ $-A_{0}^{a} G^{a}$ is used in the Lagrangian, ${ }^{2}$

$$
\mathcal{L}=-\frac{1}{4} F_{i j}^{a} F_{i j}^{a}+\frac{1}{2} F_{i 0}^{a} F_{i 0}^{a}+j_{i}^{a} A_{i}^{a}-j_{0}^{a} A_{0}^{a}+\mathcal{L}_{\text {gauge }}-\bar{\psi}(m-i \gamma \cdot \partial) \psi
$$

and the Dirac-Bergmann method of constrained quantization is applied Lagrange multiplier field $G^{a}$ is incorporated into the time-derivative of $\Pi_{0}^{a}$, which, in this case is $D_{i} \Pi_{i}^{a}+j_{0}^{a}+G^{a}$. The presence of the Lagrange multiplier field assures a second-class system of constraints, and also terminates the chain of secondary constraints very quickly. The resulting Dirac commutators differ in only trivial ways from canonical Poisson commutators. Alternatively, it is possible to entirely avoid the need to consider primary constraints in the temporal gauge by using the gauge-fixing term $-\partial_{0} A_{0}^{a} G^{a}$ instead of $-A_{0}^{a} G^{a}$, so that $-G^{a}$ becomes the momentum canonically conjugate to $A_{0}$. The gauge constraint then is $\partial_{0} A_{0}=0$, which, with the imposition of $A_{0}=0$ and Gauss's

${ }^{a}$ we use nonrelativistic notation, in which all space-time indices are subscripted and designate contravariant components of contravariant quantities such as $A_{i}^{a}$ or $j_{i}^{a}$, and covariant components of covariant quantities such as $\partial_{i}$. Repeated indices are summed from $1 \rightarrow 3$. 
law at one particylar time, implements both, the gauge condition and Gauss's law for all times 3 The same procedure can be extended to all axial gauges for which the gauge condition is $A_{0}^{a}+\gamma A_{3}^{a}=0$, where $\gamma$ is a variable real parameter 4 . Finally, a very direct way of quantizing QCP in the temporal gauge is simply to set $A_{0}^{a}=0$ in the original Lagrangian 10 ,

In the process of quantizing QCD in the temporal gauge by any of the procedures outlined above, The Gribov ambiguity does not arise. In contrast, the Gribov ambiguity impedes the canonical quantization of QCD in the Coulomb gauge. The inverse of the matrix of constraint commutators - required for the implementation of the Dirac-Bergmann procedure - depends on the inverse of the Faddeev-Popov operator $(D \cdot \partial)^{a b}=\left(\delta_{a b} \partial^{2}+g \epsilon^{a u b} A_{n}^{u} \partial_{n}\right)$. In the Coulomb gauge, the inverse of $(D \cdot \partial)^{a b}$ is not unique, and therefore the inverse of the matrix of constraint commutators does not exist. In this way, the existence of Gribov copies of the Coulomb-gauge field impedes the quantization of QCD in that gauge; but quantization of QCD in the temporal gauge is not similarly affected by this difficulty. It is on this basis that some authors have concluded that there is no Gribov ambiguity in the temporal gauge 8; a variety of other arguments have also been put forth to support the conclusion that axial gauges have no Gribov copies 9 .

It is significant that when QCD is quantized in the temporal gauge, Gauss's law is not implemented - the imposition of the non-Abelian Gauss's law and the construction of gauge-invariant fields remains to be addressed. If it were possible to use the Dirac-Bergmann procedure consistently to quantize QCD in the Coulomb gauge, the implementation of Gauss's law would be an inevitable consequence - it would appear as one of the Dirac-Bergmann secondary constraints (specifically from the Poisson bracket of the total Hamiltonian with the gauge condition $\left.\partial_{i} A_{i}^{a}=0\right)$. The procedure would then lead to a Hamiltonian expressed in terms of unconstrained, independent field variables. As it is, however, the quantization of QCD in the Coulomb gauge is very problematical for a number of reasons - Gribov copies and ordering ambiguities of non-commuting operators, among them.

In our work, we have shown how the temporal-gauge formulation of QCD can be brought into compliance with Gauss's law, by explicitly constructing a set of state vectors that are annihilated by the Gauss's law operator

$$
\hat{\mathcal{G}}^{a}(\mathbf{r})=\overbrace{\partial_{i} \Pi_{i}^{a}(\mathbf{r})+\underbrace{D_{i} \Pi_{i}^{a}(\mathbf{r}) \equiv \mathcal{G}^{a}(\mathbf{r})}_{J_{0}^{a}(\mathbf{r})} f^{a b c} A_{i}^{b}(\mathbf{r}) \Pi_{i}^{c}(\mathbf{r})}+j_{0}^{a}(\mathbf{r}),
$$

where $j_{0}^{a}(\mathbf{r})$ is the quark color-charge density $j_{0}^{a}(\mathbf{r})=g \psi^{\dagger}(\mathbf{r}) \frac{\lambda^{a}}{2} \psi(\mathbf{r}) ; J_{0}^{a}(\mathbf{r})$ is 
the glue color-charge density as well as part of the covariant derivative of $\Pi_{i}^{a}$, which is the negative chromoelectric field and also is canonically conjugate to the gauge field $A_{i}^{a}$. The state vectors that are annihilated by $\hat{\mathcal{G}}^{a}$ comprise a space in which the time evolution of the theory takes place. In this way, the validity of Gauss's law within the framework of temporal-gauge QCD is assured.

\section{Gauss's Law and Gauge-Invariant Quark and Gluon Fields}

Gauss's law in temporal-gauge QCD is implemented by constructing state vectors $\Psi|\phi\rangle$ for which $\left\{\partial_{i} \Pi_{i}^{a}(\mathbf{r})+J_{0}^{a}(\mathbf{r})\right\} \Psi|\phi\rangle=0$, where $|\phi\rangle$ is the state annihilated by the Abelian part of $D_{i} \Pi_{i}^{a}$, so that $\partial_{i} \Pi_{i}^{a}|\phi\rangle=0$ and $\Psi$ is an operator that essentially converts $D_{i} \Pi_{i}^{a}$ to $\partial_{i} \Pi_{i}^{a}$ within the space of $|\phi\rangle$ states 10.11 . $\Psi$ must obey the operator-valued differential equation

$$
\begin{aligned}
& \left\{\partial_{i} \Pi_{i}^{a}(\mathbf{r})+J_{0}^{a}(\mathbf{r})\right\} \Psi|\phi\rangle=\Psi \partial_{i} \Pi_{i}^{a}(\mathbf{r})|\phi\rangle \\
& \text { or }\left[\partial_{i} \Pi_{i}^{a}(\mathbf{r}), \Psi\right]=-J_{0}^{a}(\mathbf{r}) \Psi+B_{Q}^{a}(\mathbf{r}),
\end{aligned}
$$

where $B_{Q}^{a}(\mathbf{r})$ is an operator that has $\partial_{i} \Pi_{i}^{a}(\mathbf{r})$ on its extreme right so that $B_{Q}^{a}(\mathbf{r})|\phi\rangle=0$. The solution of Eq. (4) has been given as 4

$$
\Psi=\|\exp (\mathcal{A})\|
$$

in which

$$
\mathcal{A}=i \int d \mathbf{r} \overline{\mathcal{A}_{i}^{\gamma}}(\mathbf{r}) \Pi_{i}^{\gamma}(\mathbf{r})
$$

and $\overline{\mathcal{A}_{i}^{\gamma}}(\mathbf{r})$ is the Resolvent Field. The resolvent field is central to this work, and largely determines the properties of gauge-invariant fields and of QCD represented in terms of those gauge-invariant fields. The $\|$ ||-ordered product orders $\Psi$ so that all functionals of $A_{i}^{a}$ are to the left of all functionals of $\Pi_{j}^{b}$; this ordering is important because, in the temporal gauge,

$$
\left[A_{i}^{a}(\mathbf{x}), \Pi_{j}^{b}(\mathbf{y})\right]=i \delta_{a b} \delta_{i j} \delta(\mathbf{x}-\mathbf{y}) .
$$

We have shown that the resolvent field obeys the integral equation 11

$$
\int d \mathbf{r} \overline{\mathcal{A}_{j}^{\gamma}}(\mathbf{r}) V_{j}^{\gamma}(\mathbf{r})=\sum_{\eta=1}^{\infty} \frac{i g^{\eta}}{\eta !} \int d \mathbf{r}\left\{\psi_{(\eta) j}^{\gamma}(\mathbf{r})+f_{(\eta)}^{\vec{\alpha} \beta \gamma} \mathcal{M}_{(\eta)}^{\vec{\alpha}}(\mathbf{r}) \overline{\mathcal{B}_{(\eta) j}^{\beta}}(\mathbf{r})\right\} V_{j}^{\gamma}(\mathbf{r})
$$

in which $\mathcal{M}_{(\eta)}^{\vec{\alpha}}(\mathbf{r})=\prod_{m=1}^{\eta} \overline{\mathcal{Y}^{\alpha[m]}}(\mathbf{r}), \overline{\mathcal{Y}^{\alpha}}(\mathbf{r})=\frac{\partial_{j}}{\partial^{2}} \overline{\mathcal{A}_{j}^{\alpha}}(\mathbf{r})$, and $\overline{\mathcal{B}_{(\eta) i}^{\beta}}(\mathbf{r})=$ $a_{i}^{\beta}(\mathbf{r})+\left(\delta_{i j}-\frac{\eta}{(\eta+1)} \frac{\partial_{i} \partial_{j}}{\partial^{2}}\right) \overline{\mathcal{A}_{i}^{\beta}}(\mathbf{r})$, thus producing the nonlinearity in Eq. (8) . 
$\psi_{(\eta) i}^{\gamma}(\mathbf{r})$ is taken as the "source" term $\psi_{(\eta) i}^{\gamma}(\mathbf{r})=(-1)^{\eta-1} f_{(\eta)}^{\vec{\alpha} \beta \gamma} \mathcal{R}_{(\eta)}^{\vec{\alpha}}(\mathbf{r}) \mathcal{Q}_{(\eta) i}^{\beta}(\mathbf{r})$, where $\mathcal{R}_{(\eta)}^{\vec{\alpha}}(\mathbf{r})=\prod_{m=1}^{\eta} \mathcal{X}^{\alpha[m]}(\mathbf{r}), \mathcal{X}^{\alpha}(\mathbf{r})=\frac{\partial_{j}}{\partial^{2}} A_{j}^{\alpha}(\mathbf{r})$, and $\mathcal{Q}_{(\eta) i}^{\beta}(\mathbf{r})=\left[a_{i}^{\beta}(\mathbf{r})+\right.$ $\left.\frac{\eta}{(\eta+1)} x_{i}^{\beta}(\mathbf{r})\right] ; a_{i}^{\gamma}(\mathbf{r})$ and $x_{i}^{\gamma}(\mathbf{r})$ are the transverse and longitudinal parts of the gauge field respectively, and $f_{(\eta)}^{\vec{\alpha} \beta \gamma}$ denotes the chain of structure constants

$$
f_{(\eta)}^{\vec{\alpha} \beta \gamma}=f^{\alpha[1] \beta b[1]} f^{b[1] \alpha[2] b[2]} f^{b[2] \alpha[3] b[3]} f^{b[\eta-2] \alpha[\eta-1] b[\eta-1]} f^{b[\eta-1] \alpha[\eta] \gamma} .
$$

It is an interesting fact that Eq. (8) for the $\mathrm{SU}(2)$ case takes a form strongly reminiscent of a finite gauge transformation for fields in an adjoint representation of $\mathrm{SU}(2) 11$.

$\hat{\mathcal{G}}^{a}$ and $\mathcal{G}^{a}=D_{i} \Pi_{i}^{a}$ are unitarily equivalent, and are related by

$$
\hat{\mathcal{G}}^{a}(\mathbf{r})=\mathcal{U}_{\mathcal{C}} \mathcal{G}^{a}(\mathbf{r}) \mathcal{U}_{\mathcal{C}}^{-1}
$$

where $\mathcal{U}_{\mathcal{C}}=e^{\mathcal{C}_{0}} e^{\overline{\mathcal{C}}}$ and where $\mathcal{C}_{0}=i \int d \mathbf{r} \mathcal{X}^{\alpha}(\mathbf{r}) j_{0}^{\alpha}(\mathbf{r})$ and $\overline{\mathcal{C}}=i \int d \mathbf{r} \overline{\mathcal{Y}^{\alpha}}(\mathbf{r}) j_{0}^{\alpha}(\mathbf{r})$. With this unitary equivalence, $\mathcal{G}^{a}$ can be used to represent $\hat{\mathcal{G}}^{a}$ in a new representation, which we will call the $\mathcal{N}$ representation. In this representation, the quark field $\psi$ is gauge-invariant because it commutes with $\mathcal{G}^{a}$. This unitary equivalence can be used to construct gauge invariant operator-valued quark fields in the original representation (which we call the $\mathcal{C}$ representation):

$$
\begin{gathered}
\psi_{\mathrm{GI}}(\mathbf{r})=\mathcal{U}_{\mathcal{C}} \psi(\mathbf{r}) \mathcal{U}_{\mathcal{C}}^{-1} \text { and } \psi_{\mathrm{GI}}^{\dagger}(\mathbf{r})=\mathcal{U}_{\mathcal{C}} \psi^{\dagger}(\mathbf{r}) \mathcal{U}_{\mathcal{C}}^{-1} \\
\text { or, equivalently, } \quad \psi_{\mathrm{GI}}(\mathbf{r})=V_{\mathcal{C}}(\mathbf{r}) \psi(\mathbf{r}) \quad \text { and } \quad \psi_{\mathrm{GI}}^{\dagger}(\mathbf{r})=\psi^{\dagger}(\mathbf{r}) V_{\mathcal{C}}^{-1}(\mathbf{r}), \\
\text { where } V_{\mathcal{C}}(\mathbf{r})=\exp \left(-i g \overline{\mathcal{Y}^{\alpha}}(\mathbf{r}) \frac{\lambda^{\alpha}}{2}\right) \exp \left(-i g \mathcal{X}^{\alpha}(\mathbf{r}) \frac{\lambda^{\alpha}}{2}\right)
\end{gathered}
$$

The transformation of $\psi$ to the gauge-invariant $\psi_{\mathrm{GI}}(\mathbf{r})=V_{\mathcal{C}}(\mathbf{r}) \psi(\mathbf{r})$ has the formal structure of a gauge transformation, because $V_{\mathcal{C}}$ can be written as

$$
V_{\mathcal{C}}=\exp \left[-i g \mathcal{Z}^{\alpha} \frac{\lambda^{\alpha}}{2}\right]=\exp \left[-i g \overline{\mathcal{Y}}^{\alpha} \frac{\lambda^{\alpha}}{2}\right] \exp \left[-i g \mathcal{X}^{\alpha} \frac{\lambda^{\alpha}}{2}\right] .
$$

However, in fact, under a gauge transformation, $V_{\mathcal{C}}$ and $\psi \underline{\text { both }}$ are transformed, so that

$$
\psi \rightarrow \exp \left(-i \omega^{\gamma} \frac{\lambda^{\gamma}}{2}\right) \psi \quad \text { and } \quad V_{\mathcal{C}} \rightarrow V_{\mathcal{C}} \exp \left(i \omega^{\gamma} \frac{\lambda^{\gamma}}{2}\right)
$$

and $\psi_{\mathrm{GI}}(\mathbf{r})$ remains strictly gauge invariant. Furthermore, the formal similarity of $V_{\mathcal{C}}$ to a gauge transformation enables us to construct gauge-invariant gluon fields

$$
A_{\mathrm{Gl} i}^{b}(\mathbf{r}) \frac{\lambda^{b}}{2}=V_{\mathcal{C}}(\mathbf{r})\left[A_{i}^{b}(\mathbf{r}) \frac{\lambda^{b}}{2}\right] V_{\mathcal{C}}^{-1}(\mathbf{r})+\frac{i}{g} V_{\mathcal{C}}(\mathbf{r}) \partial_{i} V_{\mathcal{C}}^{-1}(\mathbf{r})
$$


which is equivalent to $A_{\mathrm{Gl} i}^{b}(\mathbf{r})=A_{T i}^{b}(\mathbf{r})+\left[\delta_{i j}-\frac{\partial_{i} \partial_{j}}{\partial^{2}}\right] \overline{\mathcal{A}_{i}^{b}}(\mathbf{r})$,

so that $A_{\mathrm{Gl} i}^{b}$ turns out to be transverse - - the sum of the transverse parts of the gauge field and the resolvent field.

We have been able to express the QCD Hamiltonian entirely in terms of gauge-invariant field operators 14, 15,16 . For that purpose, we have defined gauge-invariant versions of $\Pi_{i}^{a}$ and of the Gauss's law operator. These quantities are $\Pi_{\mathrm{GI} i}^{b}=\mathcal{R}_{b d} \Pi_{i}^{d}$ and $\mathcal{G}_{\mathrm{GI}}^{b}=\mathcal{R}_{b d} \mathcal{G}^{d}$ respectively, where $\mathcal{R}_{b d}=\frac{1}{2} \operatorname{Tr}\left[V_{\mathcal{C}}^{-1} \lambda^{b} V_{\mathcal{C}} \lambda^{d}\right]$. This QCD Hamiltonian (in the $\mathcal{N}$ representation, in which $\psi$ is the gaugeinvariant form of the quark field) is

$$
\begin{aligned}
H_{\mathcal{N}}= & \int d \mathbf{r}\left\{\frac{1}{2} \Pi_{\mathrm{Gl} i}^{a}(\mathbf{r}) \Pi_{\mathrm{Gl} i}^{a}(\mathbf{r})+\frac{1}{4} F_{\mathrm{Gl} i j}^{a}(\mathbf{r}) F_{\mathrm{Gl} i j}^{a}(\mathbf{r})+\right. \\
& \left.\psi^{\dagger}(\mathbf{r})\left[\beta m-i \alpha_{i}\left(\partial_{i}-i g A_{\mathrm{Gl} i}^{a}(\mathbf{r}) \frac{\lambda^{\alpha}}{2}\right)\right] \psi(\mathbf{r})\right\}+\tilde{H}_{L R}+\tilde{H}_{\mathcal{G}}
\end{aligned}
$$

where

$$
\begin{gathered}
\tilde{H}_{L R}=\int d \mathbf{r}\left(\frac{1}{2} J_{0(\mathrm{Gl})}^{a \dagger} \frac{1}{\partial^{2}} \mathcal{K}_{0}^{a}(\mathbf{r})+\frac{1}{2} \mathcal{K}_{0}^{a}(\mathbf{r}) \frac{1}{\partial^{2}} J_{0(\mathrm{Gl})}^{a}-\frac{1}{2} \mathcal{K}_{0}^{a}(\mathbf{r}) \frac{1}{\partial^{2}} \mathcal{K}_{0}^{a}(\mathbf{r})\right) \\
\text { and } H_{\mathcal{G}}=-\frac{1}{2} \int d \mathbf{r}\left[\mathcal{G}_{\mathrm{GI}}^{a}(\mathbf{r}) \frac{1}{\partial^{2}} \mathcal{K}_{0}^{a}(\mathbf{r})+\mathcal{K}_{0}^{a}(\mathbf{r}) \frac{1}{\partial^{2}} \mathcal{G}_{\mathrm{GI}}^{a}(\mathbf{r})\right]
\end{gathered}
$$

$J_{0(\mathrm{Gl})}^{a}$ is the gauge-invariant glue color charge density $J_{0(\mathrm{Gl})}^{a}=g f^{a b c} A_{\mathrm{Gl} i}^{b} \Pi_{\mathrm{Gl} i}^{c}$, $F_{\mathrm{Gl} i j}^{a}$ is the gauge-invariant chromomagnetic field in which $A_{\mathrm{Gl} i}^{a}$ replaces the gauge-dependent $A_{i}^{a}$, and $\mathcal{K}_{0}^{a}$ is a gauge-invariant effective quark color charge density, given by

$$
\left(\delta_{a b}+g f^{a u b} A_{\mathrm{Gl}}^{u} \frac{\partial_{i}}{\partial^{2}}\right) \mathcal{K}_{0}^{b}=-j_{0}^{a} \text { for } j_{0}^{a}=g \psi^{\dagger} \frac{\lambda^{a}}{2} \psi .
$$

The relation of $H_{\mathcal{N}}$ to the Coulomb-gauge formulation of QCD is of particular interest. We first note the following commutation relations: 16

$$
\left[\Pi_{\mathrm{Gl} j}^{b}(\mathbf{y}), A_{\mathrm{Gl} i}^{a}(\mathbf{x})\right]=-i\left\{\delta_{a b} \delta(\mathbf{y}-\mathbf{x})-\partial_{j} \mathcal{D}^{b h}(\mathbf{y}, \mathbf{x}) \overleftarrow{L}_{i}^{h a}(\mathbf{x})\right\}
$$

where $\mathcal{D}^{b h}(\mathbf{y}, \mathbf{x})$ designates the formal series representation of the inverse of the Faddeev-Popov operator, and the arrow indicates action to the left; and

$$
\left[\Pi_{\mathrm{Gl} i}^{\alpha}(\mathbf{x}), \Pi_{\mathrm{Gl} j}^{\beta}(\mathbf{y})\right]=i g\left\{\partial_{i} \mathcal{D}^{\alpha h}(\mathbf{x}, \mathbf{y}) \epsilon^{h \gamma \beta} \Pi_{\mathrm{Gl} j}^{\gamma}(\mathbf{y})-\partial_{j} \mathcal{D}^{\beta h}(\mathbf{y}, \mathbf{x}) \epsilon^{h \gamma \alpha} \Pi_{\mathrm{Gl} i}^{\gamma}(\mathbf{x})\right\} .
$$

${ }^{b}$ Iterative expansions of these gauge-invariant quark and gluonffites agree with the iterative representations of these fields given by Lavelle and McMullan 12.13. 
These commutation rules agree with those given by Schwinger for QCP in the Coulomb gauge, modulo operator order in corresponding expressions 17 . The Hamiltonian $\left(H_{\mathcal{N}}-H_{\mathcal{G}}\right)$ also has many features in common with the Hamiltonian in earlier work on Coulomb-gauge QCD by Christ and Lee18, by Creutz et. al $2 \mathrm{~g}$, and by Gervais and Sakita 22. A $H_{\mathcal{N}}$ however is not the Coulombgauge Hamiltonian. $H_{\mathcal{G}}$ "remembers" that the Hamiltonian is in the temporal gauge; but, as we have shown elsewhere 16 , $H_{\mathcal{G}}$ can neither affect the time evolution of state vectors within the space of states annihilated by the Gauss's law operator, nor can time evolution transport state vectors out of that space. The situation in QCD is, therefore, remarkably like that in QED. When Gauss's law is imposed and the Hamiltonian is expressed in terms of physical variables, the time-evolution mediated by the temporal-gauge Hamiltonian is essentially that expected in the Coulomb-gauge (with the proviso that the latter is well-defined for QED but much less well-defined for QCD), but, because of the inclusion of $H_{\mathcal{G}}$ (or its QED equivalent), the Hamiltonian remains in its original (in this case, temporal) gauge 2122.

Even though $H_{\mathcal{N}}$ is a functional of gauge-invariant - physical - operatorvalued variables only, complete control over its properties is retained, because all gauge-invariant quantities are defined explicitly in terms of the gaugedependent $A_{i}^{a}$ and $\Pi_{j}^{b}$, whose commutation rules are the canonical ones given in Eq. (7). The suggestive position in Eq. (19) occupied by the effective colorcharge density $\mathcal{K}_{0}^{a}$ makes it important to investigate its properties in depth.

\section{Topology and the Gribov Ambiguity}

In earlier work, we have represented $\mathrm{SU}(2)$ versions of $\overline{\mathcal{A}_{i}^{\gamma}}(\mathbf{r})$ and $A_{i}^{\gamma}(\mathbf{r})$ as $c$ number functions of spatial variables - as second-rank tensors in the combined spatial and $\mathrm{SU}(2)$ indices $i$ and $\gamma$ respectively, so that, except in so far as the forms of $\overline{\mathcal{A}_{i}^{\gamma}}(\mathbf{r})$ and $A_{i}^{\gamma}(\mathbf{r})$ must reflect this second-rank tensor structure, they are isotropic functions of the position 23 . With this representation we obtain

$$
\begin{gathered}
\overline{\mathcal{A}}_{i}^{\gamma}(\mathbf{r})=\frac{1}{g}\left[\left(\delta_{i \gamma}-\frac{r_{i} r_{\gamma}}{r^{2}}\right) \frac{\overline{\mathcal{N}}(r)}{r}+\frac{r_{i} r_{\gamma}}{r^{2}} \overline{\mathcal{N}}^{\prime}(r)\right], \\
\overline{\mathcal{A}}_{i}^{\gamma}(\mathbf{r})=\delta_{i \gamma} \varphi_{A}(r)+\frac{r_{i} r_{\gamma}}{r^{2}} \varphi_{B}(r)+\epsilon_{i \gamma n} \frac{r_{n}}{r} \varphi_{C}(r), \text { as well as } \\
A_{i}^{\gamma}{ }^{L}(\mathbf{r})=\frac{1}{g}\left[\left(\delta_{i \gamma}-\frac{r_{i} r_{\gamma}}{r^{2}}\right) \frac{\mathcal{N}(r)}{r}+\frac{r_{i} r_{\gamma}}{r^{2}} \mathcal{N}^{\prime}(r)\right] \text { and }
\end{gathered}
$$

${ }^{c}$ Note, however, that the chromoelectric field in these works are taken to be purely transverse. 


$$
A_{i}^{\gamma T}(\mathbf{r})=\delta_{i \gamma} \mathcal{T}_{A}(r)+\frac{r_{i} r_{\gamma}}{r^{2}} \mathcal{T}_{B}(r)+\epsilon_{i \gamma n} \frac{r_{n}}{r} \mathcal{T}_{C}(r),
$$

where, to preserve transversality in the transverse fields,

$$
\left(r^{2} \varphi_{B}\right)^{\prime}+r^{2}\left(\varphi_{A}\right)^{\prime}=0 \text { and }\left(r^{2} \mathcal{T}_{B}\right)^{\prime}+r^{2}\left(\mathcal{T}_{A}\right)^{\prime}=0 .
$$

With this representation, $A_{\mathrm{Gl} i}^{\gamma}(\mathbf{r})$ becomes

$$
\begin{aligned}
& A_{\mathrm{G} I}^{\gamma}(\mathbf{r})=\frac{1}{g r}\left\{\epsilon_{i \gamma n} \frac{r_{n}}{r}[\cos (\overline{\mathcal{N}}+\mathcal{N})-1+\mathcal{N} \sin (\overline{\mathcal{N}}+\mathcal{N})]+\left(\delta_{i \gamma}-\frac{r_{i} r_{\gamma}}{r^{2}}\right) \times\right. \\
& \left.\times[\mathcal{N} \cos (\overline{\mathcal{N}}+\mathcal{N})-\sin (\overline{\mathcal{N}}+\mathcal{N})]-\frac{r_{i} r_{\gamma}}{r} \frac{d \overline{\mathcal{N}}}{d r}\right\} \\
& +\mathcal{T}_{A}\left\{\left(\delta_{i \gamma}-\frac{r_{i} r_{\gamma}}{r^{2}}\right) \cos (\overline{\mathcal{N}}+\mathcal{N})+\epsilon_{i \gamma n} \frac{r_{n}}{r} \sin (\overline{\mathcal{N}}+\mathcal{N})\right\}+\frac{r_{i} r_{\gamma}}{r^{2}}\left(\mathcal{T}_{A}+\mathcal{T}_{B}\right) \\
& +\mathcal{T}_{C}\left\{\epsilon_{i \gamma n} \frac{r_{n}}{r} \cos (\overline{\mathcal{N}}+\mathcal{N})-\left(\delta_{i \gamma}-\frac{r_{i} r_{\gamma}}{r^{2}}\right) \sin (\overline{\mathcal{N}}+\mathcal{N})\right\} .
\end{aligned}
$$

When Eqs. (24 -27) are substituted into the integral equation - Eq. (8) - for the $\mathrm{SU}(2)$ case, it becomes a nonlinear differential equation in which $\overline{\mathcal{N}}(r)$ is the dependent function of $r$, and the other functions are source terms. When the independent variable is changed to $u=\ln \left(\frac{r}{r_{0}}\right)$, this equation becomes

$$
\begin{aligned}
\frac{d^{2} \overline{\mathcal{N}}}{d u^{2}} & +\frac{d \overline{\mathcal{N}}}{d u}+2[\mathcal{N} \cos (\overline{\mathcal{N}}+\mathcal{N})-\sin (\overline{\mathcal{N}}+\mathcal{N})] \\
& +2 g r_{0} \exp (u)\left\{\mathcal{T}_{A}[\cos (\overline{\mathcal{N}}+\mathcal{N})-1]-\mathcal{T}_{C} \sin (\overline{\mathcal{N}}+\mathcal{N})\right\}=0
\end{aligned}
$$

Since $\overline{\mathcal{N}}(r)$ must be bounded everywhere in $0 \leq r<\infty, \overline{\mathcal{N}}(u)$ must be bounded everywhere in $-\infty<r<\infty$. Studies have shown that, for the identical source terms $\mathcal{N}, \mathcal{T}_{A}$, and $\mathcal{T}_{C}$, there are a number of different solutions of this equation, and they define different topological sectors of the gauge theory 23 . Although $A_{\mathrm{Gl} i}^{\gamma}(\mathbf{r})$ is invariant to "small" gauge transformations, which are continuously deformable to the identity, they can exist in different topological sectors which are connected by "large" gauge transformations. The different topological sectors manifested when Eq. (29) is solved, reflect the existence of these large gauge transformations. When the gauge field vanishes, i.e, $A_{i}^{\gamma}(\mathbf{r})=0$, the differential equation reduces to the autonomous equation

$$
\frac{d^{2} \overline{\mathcal{N}}}{d u^{2}}+\frac{d \overline{\mathcal{N}}}{d u}-2 \sin (\overline{\mathcal{N}})=0
$$

which is the equation for a damped pendulum with large amplitude vibrations (also: Gribov equation) with the following caveat: For the pendulum, $u$ is the 
time, and $\overline{\mathcal{N}}$ for that case is bounded for $0 \leq u<\infty$. The equation then has multiple solutions, corresponding to multiple turns and swings executed before the pendulum comes to rest at equilibrium. For the gauge theory, $u=\ln \left(\frac{r}{r_{0}}\right)$, and $\overline{\mathcal{N}}$ for that case is bounded for $-\infty<u<\infty$. The equation now does not have multiple solutions and there are no Gribov copies 24 .

Gribov discussed the case of a transverse $\mathrm{SU}(2)$ gauge field

$$
\mathrm{A}_{i}=\mathrm{A}_{i}^{c} \frac{\tau^{c}}{2} \text { with } \mathrm{A}_{i}^{c}=\epsilon^{i j c} \frac{r_{j}}{r^{2}} f(r),
$$

gauge-transformed to

$$
\mathrm{A}_{i}^{\prime}=U \mathrm{~A}_{i} U^{-1}+i U \partial_{i} U^{-1} \text { where } U=\exp \left(-i \phi(r) \frac{\vec{r} \cdot \vec{\tau}}{2 r}\right) .
$$

The condition that $\mathrm{A}_{i}^{\prime}$ is also transverse, leads to

$$
\frac{d^{2} \phi}{d u^{2}}+\frac{d \phi}{d u}-2 \sin (\phi)(1-f(u))=0
$$

$f(u)$ keeps Eq. (33) from being autonomous, and it has multiple solutions for the same $f(u)$, as does our Eq. (29). We draw the following conclusions from these observations:

- When QCD is quantized in the Coulomb gauge, the quantization procedure is impeded by the existence of Gribov copies, because the non-uniqueness of the inverse of the faddeev-Popov operator prevents the inversion of the Dirac constrained commutator matrix.

- When the quantization is carried out in the temporal gauge, no impediments to the inversion of the commutator matrix arise, and the procedure can easily be carried out in a variety of ways. But, in contrast to the Coulomb gauge, Gauss's law is not implemented in the process.

- Gribov copies arise in the temporal gauge when Gauss's law is implemented. It is the imposition of gauge invariance and the imposition of Gauss's law that generates Gribov copies in QCD. This is consistent with the theorem proven by Singer 25 .

\section{Acknowledgments}

This research was supported by the Department of Energy under Grant No. DE-FG02-92ER40716.00. 


\section{References}

1. P. A. M. Dirac, Lectures on Quantum Mechanics (Yeshiva University Press, New York, 1964); also see P. A. M. Dirac, Can. J. Math. 2 (1950) 129.

2. P. G. Bergmann and I. Goldberg, Phys. Rev. 98 (1955) 531.

3. K. Haller, Phys. Rev. D 36 (1987) 1839.

4. K. Haller, Phys. Lett. B251 (1990) 575.

5. J. Goldstone and R. Jackiw, Phys. Lett. B74 (1978) 81.

6. R. Jackiw, Rev. Mod. Phys. 52 (1980) 661.

7. G. C. Rossi and M. Testa, Nucl. Phys. B163 (1980) 109;

8. For example, S. Weinberg, The Quantum Theory of Fields Vol. II (Cambridge Univ. Press, Cambridge, UK, 1995), Section 15.4.

9. For example, A. Bassetto, G Nardelli and R. Soldati, Yang-Mills Theories in Algebraic Non-Covariant Gauges (World Scientific, Singapore 1991).

10. M. Belloni, L. Chen and K. Haller, Phys. Lett. B373 (1996) 185.

11. L. Chen, M. Belloni and K. Haller, Phys. Rev. D 55, 2347 (1997).

12. M. Lavelle and D. McMullan, Phys. Lett. B329 (1994) 68.

13. M. Lavelle and D. McMullan, Phys. Rept. 279 (1997) 1.

14. M. Belloni, L. Chen and K. Haller, Phys. Lett. B403 (1997) 316.

15. L. Chen and K. Haller, Int. J. Mod. Phys. A14 (1999) 2745.

16. K Haller, Int. J. Mod. Phys. A16 (2001) 2789.

17. J. Schwinger, Phys. Rev. 125 (1962) 1043.

18. N. H. Christ and T. D. Lee, Phys. Rev. D 22 (1980) 939.

19. M. Creutz, I. J. Muzinich, and T. N. Tudron, Phys. Rev. D 19 (1979) 531.

20. J. L. Gervais and B. Sakita, Phys. Rev. D 18 (1978) 453.

21. K. Haller, Phys. Rev. D 36 (1987) 1830.

22. K. Haller and E. Lim-Lombridas, Foundations of Physics 24 (1994) 217.

23. K. Haller, L. Chen and Y. S. Choi, Phys. Rev. D 60 (1999) 125010.

24. V. N. Gribov, Instability of non-Abelian gauge theories and impossibility of choice of Coulomb gauge, Lecture at the 12th Winter School of the Leningrad Nuclear Physics Institute (unpublished).

25. I. M. Singer, Commun. Math. Phys. 60 (1978) 7. 